\title{
On Double Danielewski Surfaces and the Cancellation Problem
}

\author{
Neena Gupta* and Sourav Sen ${ }^{\dagger}$ \\ * Stat-Math Unit, Indian Statistical Institute, \\ 203 B.T. Road, Kolkata 700 108, India. \\ e-mail : neenag@isical.ac.in \\ ${ }^{\dagger}$ Swami Vivekananda Research Centre, \\ Ramakrishna Mission Vidyamandira \\ P.O. Belur Math, Howrah 711202, India. \\ e-mail : sourav.sen3@gmail.com
}

\begin{abstract}
We study a two-dimensional family of affine surfaces which are counter-examples to the Cancellation Problem. We describe the Makar-Limanov invariant of these surfaces, determine their isomorphism classes and characterize the automorphisms of these surfaces.

Keywords. Cancellation Problem, Exponential Maps, Makar-Limanov Invariant, Automorphism, Stable Isomorphism.

2010 MSC. Primary: 14R05, 14R10; Secondary: 13A50, 13B25, 13A02, 14 R20.

\section{Introduction}

For integral domains $C \subset A$, the notation $A=C^{[n]}$ will mean that $A=C\left[t_{1}, \ldots, t_{n}\right]$ for elements $t_{1}, \ldots, t_{n} \in A$ algebraically independent over $C$.

Let $k$ be a field. A version of the Cancellation Problem asks:

Question 1: If $A$ and $B$ are two finitely generated $k$-algebras such that $A^{[1]} \cong_{k} B^{[1]}$, does this necessarily imply that $A \cong_{k} B$ ?

For a finitely generated $k$-algebra $B$, if there exists a $k$-algebra $A$ such that $A^{[1]} \cong_{k} B^{[1]}$ but $A \varlimsup_{k} B$, then we say that $B$ does not satisfy the cancellation property.

The Cancellation Problem is known to have an affirmative solution when $\operatorname{dim} B=1$ (see [1]) but in higher dimensions there are known counter-examples to this problem. In [13], M. Hochster showed that the coordinate ring of the tangent bundle over the real sphere does not satisfy the cancellation property. However, T. Fujita, M. Miyanishi and T. Sugie $(9],[14])$ proved the cancellation property of $k^{[2]}$ for any field $k$ of
\end{abstract}


characteristic zero and P. Russell ([15]) extended their results over perfect fields of arbitrary characteristic. S.M. Bhatwadekar and the first author ([2]) established the cancellation property of $k^{[2]}$ over any arbitrary field $k$. When ch. $k>0$ and $r \geq 3$, it has been shown that $B=k^{[r]}$ does not satisfy the cancellation property (see [10], [12]). When ch. $k=0$ and $r \geq 3$, it is not known whether the polynomial $\operatorname{ring} B=k^{[r]}$ has the cancellation property.

In 1989, W. Danielewski constructed explicit examples (4]) of two-dimensional affine domains over the field of complex numbers $\mathbb{C}$ which do not satisfy the cancellation property. For any non-constant polynomial $P(Z)$ with distinct roots, Danielewski considered the coordinate ring of the affine surface $S_{n}$ defined by the equation $x^{n} y-$ $P(z)=0$ in $\mathbb{A}_{k}^{3}$. Such rings are known as Danielewski surfaces. It is known that for any pair $(m, n)$ with $m \neq n, S_{m} ¥ S_{n}$ but $S_{m} \times \mathbb{A}_{k}^{1} \cong S_{n} \times \mathbb{A}_{k}^{1}$ (cf. [7], [8, p. 246]).

In this paper, we study a family of two-dimensional affine surfaces $S_{d, e}$ over a field $k$ (of any characteristic), defined by a pair of equations

$$
\left\{x^{d} y-P(x, z)=0, x^{e} t-Q(x, y, z)=0\right\} \text { in } \mathbb{A}_{k}^{4},
$$

where $d, e \in \mathbb{N}, P(X, Z)$ is monic in $Z$ and $Q(X, Y, Z)$ is monic in $Y$ with $\operatorname{deg}_{Z} P(X, Z) \geq$ $2, \operatorname{deg}_{Y} Q(X, Y, Z) \geq 2$. We call them "double Danielewski surfaces". We first compute the ML-invariant of double Danielewski surfaces (Theorem 3.8). Next, we determine the isomorphism classes of these surfaces explicitly (Theorem 3.10) and describe a characterization of their automorphisms (Theorems 3.12 and 3.13 ). We also deduce that no double Danielewski surface is isomorphic to any Danielewski surface (Corollary 3.11). Finally, we prove a stable isomorphism property of the coordinate rings of double Danielewski surfaces under certain regularity assumptions (Theorem 3.14) and hence deduce that such rings do not satisfy the cancellation property (Corollary 3.15).

\section{Exponential maps}

In this section we recall the basics of exponential maps.

Definition. Let $k$ be a field, $A$ be a $k$-algebra and let $\phi: A \rightarrow A^{[1]}$ be a $k$-algebra homomorphism. For an indeterminate $U$ over $A$, let the notation $\phi_{U}$ denote the map $\phi: A \rightarrow A[U] . \phi$ is said to be an exponential map on $A$ if $\phi$ satisfies the following two properties:

(i) $\varepsilon_{0} \phi_{U}$ is identity on $A$, where $\varepsilon_{0}: A[U] \rightarrow A$ is the evaluation at $U=0$.

(ii) $\phi_{V} \phi_{U}=\phi_{V+U}$, where $\phi_{V}: A \rightarrow A[V]$ is extended to a homomorphism $\phi_{V}$ : $A[U] \rightarrow A[V, U]$ by setting $\phi_{V}(U)=U$.

The subring $A^{\phi}=\{a \in A \mid \phi(a)=a\}$ of $A$ is said to be the ring of invariants of $\phi$.

An exponential map $\phi$ is said to be non-trivial if $A^{\phi} \neq A$. For an affine domain $A$ over a field $k$, let $\operatorname{ExP}(A)$ denote the set of all exponential maps on $A$. The MakarLimanov invariant of $A$ is a subring of $A$ defined by

$$
\operatorname{ML}(A)=\bigcap_{\phi \in \operatorname{EXP}(A)} A^{\phi} .
$$

We summarise below some useful properties of an exponential map $\phi$ (cf. [3. p. 1291-1292] and [12, Lemma 2.8]). 
Lemma 2.1. Let $A$ be an affine domain over a field $k$. Suppose that there exists a non-trivial exponential map $\phi$ on A. Then the following statements hold:

(i) $A^{\phi}$ is factorially closed in $A$.

(ii) $A^{\phi}$ is algebraically closed in $A$.

(iii) If $x \in A$ is such that $\operatorname{deg}_{U} \phi(x)$ is of minimal positive degree, and $c$ is the leading coefficient of $U$ in $\phi(x)$, then $c \in A^{\phi}$ and $A\left[c^{-1}\right]=A^{\phi}\left[c^{-1}\right][x]$.

(iv) tr. $\operatorname{deg}_{k}\left(A^{\phi}\right)=$ tr. $\operatorname{deg}_{k}(A)-1$.

(v) If $\operatorname{tr} \cdot \operatorname{deg}_{k}(A)=1$ and $\tilde{k}$ is the algebraic closure of $k$ in $A$, then $A=\tilde{k}^{[1]}$ and $A^{\phi}=\tilde{k}$.

(vi) For any multiplicative subset $S$ of $A^{\phi} \backslash\{0\}$, $\phi$ extends to a non-trivial exponential map $S^{-1} \phi$ on $S^{-1} A$ by setting $\left(S^{-1} \phi\right)(a / s):=\phi(a) / s$ for $a \in A, s \in S$; and the ring of invariants of $S^{-1} \phi$ is $S^{-1}\left(A^{\phi}\right)$.

We recall below the concept of an admissible proper $\mathbb{Z}$-filtration on an affine domain (cf. [3]).

Definition. Let $A$ be an affine domain over a field $k$. A collection of $k$-linear subspaces $\left\{A_{n}\right\}_{n \in \mathbb{Z}}$ of $A$ is said to be a proper $\mathbb{Z}$-filtration if it satisfies the following conditions:

(i) $A_{n} \subseteq A_{n+1}$ for all $n \in \mathbb{Z}$,

(ii) $A=\bigcup_{n \in \mathbb{Z}} A_{n}$,

(iii) $\bigcap_{n \in \mathbb{Z}} A_{n}=(0)$ and

(iv) $\left(A_{n} \backslash A_{n-1}\right) \cdot\left(A_{m} \backslash A_{m-1}\right) \subseteq A_{n+m} \backslash A_{n+m-1}$ for all $n, m \in \mathbb{Z}$.

We shall call a proper $\mathbb{Z}$-filtration $\left\{A_{n}\right\}_{n \in \mathbb{Z}}$ of $A$ admissible if there exists a finite generating set $\Gamma$ of $A$ such that, for any $n \in \mathbb{Z}$ and $a \in A_{n}, a$ can be written as a finite sum of monomials in elements of $\Gamma$ and each of these monomials is an element of $A_{n}$.

Any proper $\mathbb{Z}$-filtration on $A$ determines the following $\mathbb{Z}$-graded integral domain

$$
\operatorname{gr}(A):=\bigoplus_{i} A_{i} / A_{i-1}
$$

and a map

$$
\rho: A \rightarrow \operatorname{gr}(A) \text { defined by } \rho(a)=a+A_{n-1}, \quad \text { if } a \in A_{n} \backslash A_{n-1} .
$$

An exponential map $\phi$ on a graded $\operatorname{ring} A$ is said to be homogeneous if $\phi: A \rightarrow A[U]$ becomes homogeneous when $A[U]$ is given a grading induced from $A$ such that $U$ is a homogeneous element.

Remark 2.2. Note that if $\phi$ is a homogeneous exponential map on a graded $\operatorname{ring} A$, then $A^{\phi}$ is a graded subring of $A$.

Definition 2.3. Let $A$ be an affine domain over a field $k$. A $\mathbb{Z}$-grading of $A$ is a family $\left\{A_{n}\right\}_{n \in \mathbb{Z}}$ of subgroups of $(A,+)$ such that:

(i) $A=\bigoplus_{n \in \mathbb{Z}} A_{n}$.

(ii) $A_{n} A_{m} \subseteq A_{n+m}$ for all $n, m \in \mathbb{Z}$. 
Finally, we state below a result on homogenization of exponential maps due to $\mathrm{H}$. Derksen, O. Hadas and L. Makar-Limanov ([5]) (cf. [10, Theorem 2.3]).

Theorem 2.4. Let $A$ be an affine domain over a field $k$ with an admissible proper $\mathbb{Z}$-filtration and $\operatorname{gr}(A)$ the induced $\mathbb{Z}$-graded domain. Let $\phi$ be a non-trivial exponential map on $A$. Then $\phi$ induces a non-trivial homogeneous exponential map $\bar{\phi}$ on $\operatorname{gr}(A)$ such that $\rho\left(A^{\phi}\right) \subseteq \operatorname{gr}(A)^{\bar{\phi}}$.

\section{Main Theorems}

Throughout the section $k$ will denote a field and $B$ will denote the ring

$$
B=\frac{k[X, Y, Z, T]}{\left(X^{d} Y-P(X, Z), X^{e} T-Q(X, Y, Z)\right)},
$$

where $d, e \in \mathbb{N}, P(X, Z) \in k[X, Z]$ is monic in $Z$ and $Q(X, Y, Z) \in k[X, Y, Z]$ is monic in $Y$. The letters $x, y, z$ and $t$ will denote the images of $X, Y, Z$ and $T$ respectively in $B$. Set $r:=\operatorname{deg}_{Z} P(X, Z)$ and $s:=\operatorname{deg}_{Y} Q(X, Y, Z)$. Note that when $r=1$ or $s=1$, the ring $B$ is a Danielewski surface. We call $B$ a "double Danielewski surface" if $r \geq 2$ and $s \geq 2$. For a ring $R$, the notation $R^{*}$ will denote the group of units of $R$.

We will compute $\operatorname{ML}(B)$ in Section 3.1 and discuss the isomorphism classes of double Daneilewski surfaces and characterize the automorphisms of $B$ in Section 3.2 .

\subsection{ML-invariant of $B$}

For convenience, we state below an elementary result.

Lemma 3.1. Let $R$ be an integral domain and $a, b \in R \backslash\{0\}$. If $a$ is not a zero-divisor on $R /(b)$, then $b^{n}$ is not a zero-divisor on $R /(a)$ for any integer $n \geq 1$.

Proof. If $b^{n} \alpha=a \beta$ for some $\alpha, \beta \in R$, then as $a$ is not a zero-divisor on $R /(b)$, we have $\beta \in b R$, and hence since $R$ is an integral domain, we have $b^{n-1} \alpha \in a R$. Proceeding in a similar manner, we will get that $\alpha \in a R$. Hence $b^{n}$ is not a zero-divisor on $R /(a)$.

We now recall another elementary lemma ([6, Lemma 2.4(2)]).

Lemma 3.2. Let $R$ be an integral domain and $a, b \in R \backslash\{0\}$. If $b$ is not a zero-divisor on $R /(a)$, then the ring $\frac{R[T]}{(b T-a)}$ is an integral domain.

Lemma 3.3. $B$ is an integral domain.

Proof. Let $R=\frac{k[X, Y, Z]}{\left(X^{d} Y-P(X, Z)\right)}$. Since $\left(X^{d} Y-P(X, Z)\right)$ is linear in $Y$ and $X$ does not divide $P(X, Z)$ in $k[X, Z],\left(X^{d} Y-P(X, Z)\right)$ is irreducible in the UFD $k[X, Y, Z]$. Hence, $R$ is an integral domain. We can identify $R$ as a subring of $B$, by identifying the images of $X, Y, Z$ in $R$ with $x, y, z$ in $B$. Then $B=R[T] /\left(x^{e} T-Q(x, y, z)\right)$.

Now $R /(x) \cong\left(\frac{k[Z]}{P(0, Z)}\right)[Y]$. Therefore, since $Q(X, Y, Z)$ is monic in $Y$, it follows that $Q(x, y, z)$ is not a zero-divisor on $R /(x)$. Hence by Lemma 3.1, $x^{e}$ is not a zerodivisor on $R /(Q(x, y, z))$. Therefore, by Lemma $3.2, B$ is an integral domain. 
In the next two results we show that there exists an admissible proper $\mathbb{Z}$-filtration on $B$ such that $\operatorname{gr}(B)$ is isomorphic to a special case of the $\operatorname{ring} B$ which we denote by $D$ and an admissible proper $\mathbb{Z}$-filtration on $D$ such that $\operatorname{gr}(D)$ is isomorphic to a further special case of the ring $D$ which we denote by $C$. Note that in these results $P(X, Z)$

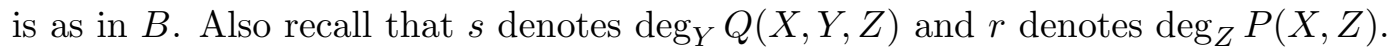

Lemma 3.4. Considering $B$ as a subring of the $\mathbb{Z}$-graded ring $k\left[x, x^{-1}, z\right]=\bigoplus_{i \in \mathbb{Z}} k[z] x^{i}$, define a proper $\mathbb{Z}$-filtration $\left\{B_{n}\right\}_{n \in \mathbb{Z}}$ on $B$ by

$$
B_{n}:=B \cap\left(\bigoplus_{i \geq-n} k[z] x^{i}\right) .
$$

This filtration on $B$ is admissible with the generating set $\{x, y, z, t\}$ and the corresponding graded ring $\operatorname{gr}(B)=\bigoplus_{n \in \mathbb{Z}}\left(B_{n} / B_{n+1}\right)$ is isomorphic to

$$
D:=\frac{k[X, Y, Z, T]}{\left(X^{d} Y-P(0, Z), X^{e} T-Y^{s}\right)} .
$$

Proof. We have $x \in B_{-1} \backslash B_{-2}, z \in B_{0} \backslash B_{-1}, y \in B_{d} \backslash B_{d-1}$ and $t \in B_{(d s+e)} \backslash B_{(d s+e-1)}$. Using the relations $x^{d} y=P(x, z)$ and $x^{e} t=Q(x, y, z)$, we see that each element $g \in B$ can be written as

$$
g=f_{0}(x, z)+\sum_{\substack{0 \leq i<d \\ j>0}} a_{i j}(z) x^{i} y^{j}+\sum_{\substack{0 \leq i<e \\ l>0}} b_{i l}(z) x^{i} t^{l}+\sum_{\substack{0 \leq i<\min \{d, e\} \\ j, l>0}} c_{i j l}(z) x^{i} y^{j} t^{l},
$$

where $f_{0}(x, z) \in k[x, z], a_{i j}(z), b_{i l}(z), c_{i j l}(z) \in k[z]$. Let $\widetilde{B}$ denote the graded ring $\operatorname{gr}(B)=\bigoplus_{n \in \mathbb{Z}}\left(B_{n} / B_{n+1}\right)$ with respect to the above filtration. For $g \in B$, let $\tilde{g}$ denote the image of $g$ in $\widetilde{B}$. It follows from (2), that the filtration defined on $B$ is admissible with the generating set $\Gamma_{\widetilde{B}}=\{x, y, z, t\}$. Hence, $\widetilde{B}$ is generated by $\tilde{x}, \tilde{y}, \tilde{z}$ and $\tilde{t}$.

We now show that, $\widetilde{B} \cong D$. Note that, $x^{d} y, P(0, z) \in B_{0}$. Hence, since $x^{d} y-$ $P(0, z) \in B_{-1}$, we have $\tilde{x}^{d} \tilde{y}-P(0, \tilde{z})=0$ in $\widetilde{B}$. Again note that $x^{e} t, y^{s} \in B_{d s}$ and $x^{e} t-y^{s}=Q(x, y, z)-y^{s} \in B_{d s-1}$. Hence, $\tilde{x}^{e} \tilde{t}-\tilde{y}^{s}=0$ in $\widetilde{B}$. As $\widetilde{B}$ can be identified with a subring of $\operatorname{gr}\left(k\left[x, x^{-1}, z\right]\right) \cong k\left[x, x^{-1}, z\right]$, we see that the elements $\tilde{x}$ and $\tilde{z}$ of $\widetilde{B}$ are algebraically independent over $k$. Since $D$ is an integral domain (cf. Lemma 3.3), we have, $\widetilde{B} \cong D$.

Lemma 3.5. Let $D$ be as in Lemma 3.4 and let $\tilde{x}, \tilde{y}, \tilde{z}, \tilde{t}$ respectively denote the images of $X, Y, Z, T$ in $D$. Considering $D$ as a subring of the $\mathbb{N}$-graded ring $k\left[\tilde{x}, \tilde{x}^{-1}, \tilde{z}\right]=$ $\bigoplus_{i \in \mathbb{N}} k\left[\tilde{x}, \tilde{x}^{-1}\right] \tilde{z}^{i}$, define a proper $\mathbb{Z}$-filtration $\left\{D_{n}\right\}_{n \in \mathbb{Z}}$ on $D$ by

$$
D_{n}:=D \cap\left(\bigoplus_{i \leq n} k\left[\tilde{x}, \tilde{x}^{-1}\right] \tilde{z}^{i}\right) .
$$

This filtration on $D$ is admissible with the generating set $\{\tilde{x}, \tilde{y}, \tilde{z}, \tilde{t}\}$ and the corresponding graded ring $\operatorname{gr}(D)=\bigoplus_{n \in \mathbb{Z}}\left(D_{n} / D_{n+1}\right)$ is isomorphic to

$$
C:=\frac{k[X, Y, Z, T]}{\left(X^{d} Y-Z^{r}, X^{e} T-Y^{s}\right)} .
$$


Proof. Note that, $D_{n}=\phi$ for all $n<0, \tilde{z} \in D_{1} \backslash D_{0}, \tilde{x} \in D_{0} \backslash D_{-1}, \tilde{y} \in D_{r} \backslash D_{r-1}$ and $\tilde{t} \in D_{r s} \backslash D_{r s-1}$. Using the relations $\tilde{x}^{d} \tilde{y}=P(0, \tilde{z})$ and $\tilde{x}^{e} \tilde{t}=\tilde{y}^{s}$, we see that each element $\tilde{g} \in D$ can be written as

$$
\tilde{g}=\sum_{i=0}^{r-1}\left(\sum_{0 \leq j<s} g_{i j}(\tilde{x}) \tilde{y}^{j}+\sum_{\substack{0 \leq j<s \\ \ell>0}} h_{i j \ell}(\tilde{x}) \tilde{y}^{j} \tilde{t}^{\ell}\right) \tilde{z}^{i},
$$

where $g_{i j}(\tilde{x}), h_{i j \ell}(\tilde{x}) \in k[\tilde{x}]$. Let $\bar{D}$ denote the graded ring $\operatorname{gr}(D)=\bigoplus_{n \in \mathbb{Z}}\left(D_{n} / D_{n-1}\right)$ with respect to the above filtration. For $\tilde{g} \in D$, let $\bar{g}$ denote the image of $\tilde{g}$ in $\bar{D}$. It follows from (3) that the filtration defined on $D$ is admissible with the generating set $\Gamma^{\prime}=\{\tilde{x}, \tilde{y}, \tilde{z}, \tilde{t}\}$. Hence, $\bar{D}$ is generated by $\bar{x}, \bar{y}, \bar{z}$ and $\bar{t}$.

We now show that $\bar{D} \cong C$. Note that $\tilde{x}^{d} \tilde{y}, \tilde{z}^{r} \in D_{r}$ and $\tilde{x}^{d} \tilde{y}-\tilde{z}^{r}=P(0, \tilde{z})-\tilde{z}^{r} \in$ $D_{r-1}$. Hence, $\bar{x}^{d} \bar{y}-\bar{z}^{r}=0$ in $\bar{D}$. Again, $\tilde{x} e \tilde{t}, \tilde{y}^{s} \in D_{r s}$ and $\tilde{x}^{e} \tilde{t}-\tilde{y}^{s}=0$ in $D$. Hence, $\bar{x}^{e} \bar{t}-\bar{y}^{s}=0$ in $\bar{D}$. As $\bar{D}$ can be identified with a subring of $\operatorname{gr}\left(k\left[\tilde{x}, \tilde{x}^{-1}, \tilde{z}\right]\right) \cong k\left[\tilde{x}, \tilde{x}^{-1}, \tilde{z}\right]$, we see that the elements $\bar{x}$ and $\bar{z}$ of $\bar{D}$ are algebraically independent over $k$. Since $C$ is an integral domain (cf. Lemma 3.3), we have, $\operatorname{gr}(D)=\bar{D} \cong C$.

Lemma 3.6. Let $C$ be the integral domain defined by

$$
\frac{k[X, Y, Z, T]}{\left(X^{d} Y-Z^{r}, X^{e} T-Y^{s}\right)}, \text { where } d, e \geq 1
$$

and any one of the following holds:

$$
\begin{array}{r}
\text { either } r \geq 2 \text { and } s \geq 2 \\
\text { or } r \geq 2 \text { and } s=1 \\
\text { or } r=1, s \geq 2 \text { and } e \geq 2 .
\end{array}
$$

Let $\bar{x}, \bar{y}, \bar{z}$ and $\bar{t}$ respectively denote the images of $X, Y, Z$ and $T$ in $C$. Consider $C=\bigoplus_{i \in \mathbb{Z}} C_{i}$ as a graded subring of $k\left[\bar{x}, \bar{x}^{-1}, \bar{z}\right]$ with

$$
C_{i}=C \cap k\left[\bar{x}, \bar{x}^{-1}\right] \bar{z}^{i} \text { for each } i \geq 0 \text { and } C_{i}=0 \text { for } i<0 .
$$

Then $C^{\phi} \subseteq k[\bar{x}]$ for any non-trivial homogeneous exponential map $\phi$ on the graded ring $C$.

Proof. Let $\phi$ be a $\mathbb{Z}$-graded exponential map on $C$. We note that this grading induces a degree function on $C$, with $\operatorname{deg} \bar{x}=0, \operatorname{deg} \bar{z}=1, \operatorname{deg} \bar{y}=r$ and $\operatorname{deg} \bar{t}=r s$. Let

$$
R=\frac{k[X, Y, T]}{\left(X^{e} T-Y^{s}\right)} .
$$

We identify $R$ as a subring of $C$ identifying the images of $X, Y$ and $T$ in $R$ with $\bar{x}, \bar{y}$ and $\bar{t}$ in $C$. Note that $R \hookrightarrow \bigoplus_{i \in r \mathbb{Z}} C_{i}$. We show that $C^{\phi} \subseteq R$.

We first note that any element $f \in C$ can be uniquely written as

$$
f=\sum_{i=0}^{r-1} f_{i} \bar{z}^{i}
$$


for some $f_{i} \in R$. For if,

$$
\operatorname{deg}\left(f_{i} \bar{z}^{i}\right)=\operatorname{deg}\left(f_{j} \bar{z}^{j}\right) \text { for some } 0 \leq i, j \leq r-1,
$$

then $\operatorname{deg}\left(f_{i}\right)+i=\operatorname{deg}\left(f_{j}\right)+j$. Since $f_{i}, f_{j} \in R$, we have

$$
\operatorname{deg}\left(f_{i}\right)-\operatorname{deg}\left(f_{j}\right) \equiv 0 \bmod r
$$

i.e., $i-j \equiv 0 \bmod r$ which implies $i=j$.

Suppose, if possible, that $C^{\phi} \nsubseteq R$. Then, as $C^{\phi}$ is a graded subring of $C, f_{i} \bar{z}^{i} \in C^{\phi}$ for some $f_{i} \in R$ and $i>0$. By Lemma 2.1(i), $\bar{z} \in C^{\phi}$. Using the relations $\bar{x}^{d} \bar{y}=\bar{z}^{r}$ and $\bar{x}^{e} \bar{t}=\bar{y}^{s}$, we see that $\bar{x}, \bar{y}, \bar{t} \in C^{\phi}$, i.e., $\phi$ is trivial, which is a contradiction. Hence, $C^{\phi} \subseteq R$.

We now show that $C^{\phi} \subseteq k[\bar{x}]$. Any element $g \in R$ can be written as

$$
g=\sum_{0 \leq i<s} g_{i}(\bar{x}) \bar{y}^{i}+\sum_{\substack{i>0 \\ 0 \leq j<s}} g_{i j}(\bar{x}) \bar{t}^{i} \bar{y}^{j},
$$

for some $g_{i}, g_{i j} \in k[\bar{x}]$. Note that

$$
\operatorname{deg}\left(g_{i}(\bar{x}) \bar{y}^{i}\right)=i r<s r \text { if } i<s \text { and } \operatorname{deg}\left(g_{i j}(\bar{x}) \bar{t}^{i} \bar{y}^{j}\right)=(\text { irs }+j r) \text { if } i>0,0 \leq j<s .
$$

Thus a homogeneous element of $C$ in $R$ is of the form $g_{i}(\bar{x}) \bar{y}^{i}$ for some $0 \leq i<s$ or $g_{i j}(\bar{x}) \bar{t}^{i} \bar{y}^{j}$ for some $i>0$ and $0 \leq j<r$. As $C^{\phi}$ is a graded subring of $C$, we have either $g_{i}(\bar{x}) \bar{y}^{i} \in C^{\phi}$ for some $0 \leq i<s$ or $g_{i j}(\bar{x}) \bar{t}^{i} \bar{y}^{j}$ for some $i>0$ and $0 \leq j<r$. Suppose $g_{i j}(\bar{x}) \bar{t}^{i} \bar{y}^{j} \in C^{\phi}$ for some $i>0$ and $0 \leq j<s$. Then $C^{\phi}$ being factorially closed in $C, \bar{t} \in C^{\phi}$ and so $\phi$ extends to a non-trivial exponential map of the ring $A:=\frac{k(T)[X, Y, Z]}{\left(X^{d} Y-Z^{r}, X^{e} T-Y^{s}\right)}$ (cf. Lemma 2.1). But since one of the conditions of (4) is satisfied, the ring $A$ is a non-normal ring of dimension one. Hence $\phi$ must be a trivial map (cf. Lemma 2.1(iii)), which is a contradiction. Hence, $\bar{t} \notin C^{\phi}$.

Therefore, $g_{i}(\bar{x}) \bar{y}^{i} \in C^{\phi}$ for some $0 \leq i<s$. If $i>0$, then $C^{\phi}$ being factorially closed in $C$, we have, $\bar{y} \in C^{\phi}$. Using the relations $\bar{x}^{e} \bar{t}=\bar{y}^{s}$ and $\bar{x}^{d} \bar{y}=\bar{z}^{r}$, we get, $\bar{x}, \bar{z}, \bar{t} \in C^{\phi}$, i.e., $\phi$ is trivial, which is a contradiction. Hence $i=0$ and $C^{\phi} \subseteq k[\bar{x}]$.

Lemma 3.7. There exists a non-trivial exponential map $\phi$ on $B$ such that $B^{\phi}=k[x]$.

Proof. Consider the map $\phi: B \rightarrow B[U]$ defined by,

$$
\begin{aligned}
\phi(x) & =x, \\
\phi(z) & =z+x^{d+e} U, \\
\phi(y) & =\frac{P\left(x, z+x^{d+e} U\right)}{x^{d}}=y+U \alpha(x, z, U), \\
\phi(t) & =\frac{Q\left(x, y+U \alpha(x, z, U), z+x^{d+e} U\right)}{x^{e}}=t+U \beta(x, y, z, U),
\end{aligned}
$$

where $\alpha(x, z, U) \in k[x, z, U], \beta(x, y, z, U) \in k[x, y, z, U]$. It is easy to see that $\phi$ is an exponential map on $B$. Clearly $k[x] \subseteq B^{\phi}$. Since $k[x]$ is algebraically closed in $B$ and tr. $\operatorname{deg}_{k[x]} B=1$, using Lemma 2.1, we see that $B^{\phi}=k[x]$. 
Theorem 3.8. Let $B$ be as in (1) and let the parameters $r, s$ and $e$ in $B$ satisfy the conditions (4) of Lemma [3.6. Then $\operatorname{ML}(B)=k[x]$.

Proof. We show that if $\phi$ is any non-trivial exponential map of $B$, then $B^{\phi}=k[x]$.

Let $\phi$ be a non-trivial exponential map of $B$. Consider the admissible proper $\mathbb{Z}$ filtration $\left\{B_{n}\right\}_{n \in \mathbb{Z}}$ on $B$ defined in Lemma 3.4 and let $\rho$ denote the canonical map $B \rightarrow \operatorname{gr}(B)=D$. By Theorem 2.4, $\phi$ induces a non-trivial exponential map $\tilde{\phi}$ on $D$ such that $\rho\left(B^{\phi}\right) \subseteq D^{\widetilde{\phi}}$. Let $f \in B^{\phi}$. Replacing $f$ by $f-\lambda$ for some $\lambda \in k^{*}$, we may assume that $\rho(f) \notin k$.

Again consider the admissible proper $\mathbb{Z}$-filtration $\left\{D_{n}\right\}_{n \in \mathbb{Z}}$ of $D$ defined in Lemma 3.5 and let $\bar{\rho}$ denote the canonical map $D \rightarrow \operatorname{gr}(D)=C$. By Theorem 2.4, $\tilde{\phi}$ induces a non-trivial exponential map $\bar{\phi}$ on $C$ such that $\bar{\rho}\left(D^{\tilde{\phi}}\right) \subseteq C^{\bar{\phi}}$. Therefore, $\bar{\rho}(\rho(f)) \in C^{\bar{\phi}}$. By Lemma [3.6, $C^{\bar{\phi}} \subseteq k[\bar{x}]$, where $\bar{x}$ denotes the image of $x$ in $C$. Hence $\bar{\rho}(\rho(f)) \in$ $k[\bar{x}] \subseteq C$. It follows from the filtration defined on $D$ in Lemma 3.5 and equation (3) that $\rho(f) \in k[\tilde{x}] \subseteq D$, where $\tilde{x}$ denotes the image of $x$ in $D$. Again from the filtration defined on $B$ in Lemma 3.4 and equation (2), it follows that $f \in k[x] \subseteq B$. Thus $B^{\phi} \subseteq k[x]$. Since $B^{\phi}$ is a factorially closed subring of $B$ of transcendence degree 1 over $k$, we have, $B^{\phi}=k[x]$. This being true for any non-trivial exponential map $\phi$ on $B$, we have by Lemma 3.7, $\mathrm{ML}(B)=k[x]$.

Remark 3.9. Let $B$ be as in (1) and suppose the parameters $r, s$ and $e$ in $B$ do not satisfy the conditions (4) of Lemma 3.6, i.e., either $\{r=s=1\}$ or $\{r=e=1$ and $s \geq 2\}$. If $r=s=1$, then $B \cong k^{[2]}$ and hence $\operatorname{ML}(B)=k$. If $r=e=1$ and $s \geq 2$, then $B \cong k[X, Y, Z] /(X Z-f(Y))$ for some polynomial $f(Y) \in k[Y]$. In this case also $\operatorname{ML}(B)=k$ (cf. [8, p.247]).

\subsection{Isomorphism Classes}

We now investigate isomorphism classes of a family of surfaces which includes the double Danielewski surfaces. We consider two such surfaces which, for convenience, we denote by $B_{1}$ and $B_{2}$ (not to be confused with the graded components of $B$ in Section 3.1):

$$
B_{1}=\frac{k[X, Y, Z, T]}{\left(X^{d_{1}} Y-P_{1}(X, Z), X^{e_{1}} T-Q_{1}(X, Y, Z)\right)}
$$

and

$$
B_{2}=\frac{k[X, Y, Z, T]}{\left(X^{d_{2}} Y-P_{2}(X, Z), X^{e_{2}} T-Q_{2}(X, Y, Z)\right)},
$$

where $d_{1}, e_{1}, d_{2}, e_{2} \in \mathbb{N}, P_{1}(X, Z), P_{2}(X, Z) \in k[X, Z]$ are monic polynomials in $Z$, $Q_{1}(X, Y, Z), Q_{2}(X, Y, Z) \in k[X, Y, Z]$ are monic polynomials in $Y$, with $r_{i}=\operatorname{deg}_{Z} P_{i}(X, Z)$ and $s_{i}=\operatorname{deg}_{Y} Q_{i}(X, Y, Z)$ for $i=1,2$. Let $x_{1}, y_{1}, z_{1}, t_{1}$ and $x_{2}, y_{2}, z_{2}, t_{2}$ denote the images of $X, Y, Z, T$ in $B_{1}$ and $B_{2}$ respectively. Suppose that the conditions (4) of Lemma 3.6 are satisfied by $\left(r_{i}, s_{i}, e_{i}\right)$ for $i=1,2$. Then from Theorem [3.8, $\operatorname{ML}\left(B_{i}\right)=k\left[x_{i}\right]$ for $i=1,2$.

Theorem 3.10. Suppose $B_{1} \cong B_{2}$. Then the following conditions hold:

(I) $\left(d_{1}, e_{1}, r_{1}, s_{1}\right)=\left(d_{2}, e_{2}, r_{2}, s_{2}\right)$. Let $(d, e, r, s)=\left(d_{i}, e_{i}, r_{i}, s_{i}\right)$ for $i=1,2$. 
(II) There exist $\lambda, \gamma \in k^{*}, \delta(X) \in k[X], f(X, Z) \in k[X, Z]$ and $h(X, Y, Z) \in k[X, Y, Z]$ such that

(i) $P_{2}(\lambda X, \gamma Z+\delta(X))=\tau P_{1}(X, Z)+X^{d} f(X, Z)$, where $\tau=\gamma^{r}\left(\in k^{*}\right)$. In particular, $P_{2}(0, \gamma Z+\delta(0))=\tau P_{1}(0, Z)$.

(ii) $Q_{2}(\lambda X, \nu Y+g(X, Z), \gamma Z+\delta(X))=\kappa Q_{1}(X, Y, Z)+X^{e} h(X, Y, Z)$, where $\nu=\lambda^{-d} \tau, \kappa=\nu^{s}$ and $g(X, Z)=\lambda^{-d} f(X, Z)$. In particular, $Q_{2}(0, \nu Y+g(0, Z), \gamma Z+\delta(0))=\kappa Q_{1}(0, Y, Z)$.

Moreover, if $\psi: B_{2} \rightarrow B_{1}$ is an isomorphism, then

$$
\begin{gathered}
\psi\left(x_{2}\right)=\lambda x_{1}, \psi\left(z_{2}\right)=\gamma z_{1}+\delta\left(x_{1}\right) \\
\psi\left(y_{2}\right)=\nu y_{1}+g\left(x_{1}, z_{1}\right) \text { and } \psi\left(t_{2}\right)=\lambda^{-e}\left(\kappa t_{1}+h(x, y, z)\right) .
\end{gathered}
$$

Conversely, if conditions (I) and (II) hold, then $B_{1} \cong B_{2}$.

Proof. Let $\psi: B_{1} \rightarrow B_{2}$ be a $k$-algebra isomorphism. Replacing $B_{1}$ by $\psi\left(B_{1}\right)$, we may assume that $B_{1}=B_{2}=B$. By Theorem [3.8, $\mathrm{ML}(B)=k\left[x_{1}\right]=k\left[x_{2}\right]$ and hence

$$
x_{2}=\lambda x_{1}+\mu
$$

for some $\lambda \in k^{*}, \mu \in k$ and $k\left(x_{1}\right)\left[z_{1}\right]=k\left(x_{2}\right)\left[z_{2}\right]$. Thus, since $B \cap k\left(x_{1}\right)=k\left[x_{1}\right]$, we have, $z_{2}=\gamma z_{1}+\delta$ for some $\gamma\left(x_{1}\right), \delta\left(x_{1}\right) \in k\left[x_{1}\right]$. Using symmetry, we have, $\gamma\left(x_{1}\right) \in k^{*}$, i.e.,

$$
z_{2}=\gamma z_{1}+\delta\left(x_{1}\right)
$$

for some $\gamma \in k^{*}, \delta\left(x_{1}\right) \in k\left[x_{1}\right]$. Hence,

$$
k\left[x_{1}, z_{1}\right]=k\left[x_{2}, z_{2}\right] .
$$

As $y_{2} \in B \subseteq k\left[x_{1}, x_{1}^{-1}, z_{1}\right]$, there exists an integer $n \geq 0$ such that $x_{1}^{n} y_{2} \in k\left[x_{1}, z_{1}\right]$. Therefore, since

$$
P_{2}\left(x_{2}, z_{2}\right)=x_{2}^{d_{2}} y_{2}=\left(\lambda x_{1}+\mu\right)^{d_{2}} y_{2},
$$

we have $x_{1}^{n} P_{2}\left(x_{2}, z_{2}\right) \in\left(\lambda x_{1}+\mu\right)^{d_{2}} k\left[x_{1}, z_{1}\right]$. If $\mu \neq 0$, then $\left(\lambda x_{1}+\mu\right) \mid P_{2}\left(x_{2}, z_{2}\right)$ in $k\left[x_{1}, z_{1}\right]$, i.e., $\left(\lambda x_{1}+\mu\right) \mid P_{2}\left(\lambda x_{1}+\mu, \gamma z_{1}+\delta\left(x_{1}\right)\right)$ in $k\left[x_{1}, z_{1}\right]$. Since $\lambda, \gamma \in k^{*}$, this contradicts that $P_{2}(X, Z)$ is monic in $Z$. Therefore, $\mu=0$ and

$$
x_{2}=\lambda x_{1}
$$

for some $\lambda \in k^{*}$.

We now show that $d_{1}=d_{2}$. Suppose, if possible, that $d_{1}>d_{2}$, Using (6) and (17), we have, $x_{1}^{d_{1}} B \cap k\left[x_{1}, z_{1}\right]=x_{2}^{d_{1}} B \cap k\left[x_{2}, z_{2}\right]$, i.e.,

$$
\left(x_{1}^{d_{1}}, P_{1}\left(x_{1}, z_{1}\right)\right) k\left[x_{1}, z_{1}\right]=\left(x_{2}^{d_{1}}, x_{2}^{d_{1}-d_{2}} P_{2}\left(x_{2}, z_{2}\right)\right) k\left[x_{2}, z_{2}\right] .
$$

Therefore, $P_{1}\left(x_{1}, z_{1}\right) \in\left(x_{2}^{d_{1}}, x_{2}^{d_{1}-d_{2}} P_{2}\left(x_{2}, z_{2}\right)\right) k\left[x_{2}, z_{2}\right] \subseteq x_{1} k\left[x_{1}, z_{1}\right]$, which contradicts that $P_{1}(X, Z)$ is a monic polynomial in $Z$. Hence, $d_{1} \leq d_{2}$ and by symmetry, we have

$$
d_{1}=d_{2}=d \text { say. }
$$


Thus, we have,

$$
x_{1}^{d} B \cap k\left[x_{1}, z_{1}\right]=x_{2}^{d} B \cap k\left[x_{2}, z_{2}\right],
$$

i.e.,

$$
\left(x_{1}^{d}, P_{1}\left(x_{1}, z_{1}\right)\right) k\left[x_{1}, z_{1}\right]=\left(x_{2}^{d}, P_{2}\left(x_{2}, z_{2}\right)\right) k\left[x_{2}, z_{2}\right] .
$$

Thus $P_{2}\left(x_{2}, z_{2}\right)=\tau^{\prime} P_{1}\left(x_{1}, z_{1}\right)+x_{1}^{d} f^{\prime}$ for some $\tau^{\prime}, f^{\prime} \in k\left[x_{1}, z_{1}\right]$. Since $P_{i}(X, Z)$ 's are monic in $Z$ (for $i=1,2$ ), using (5) and (7), we see that

$$
r_{1}\left(=\operatorname{deg}_{Z} P_{1}\right)=r_{2}\left(=\operatorname{deg}_{Z} P_{2}\right)=r \text { say, }
$$

and $\tau^{\prime} \equiv \gamma^{r} \bmod x_{1}^{d} k\left[x_{1}, z_{1}\right]$. Let $\tau=\gamma^{r}\left(\in k^{*}\right)$. Replacing $\tau^{\prime}$ by $\tau$, we have,

$$
P_{2}\left(x_{2}, z_{2}\right)=\tau P_{1}\left(x_{1}, z_{1}\right)+x_{1}^{d} f\left(x_{1}, z_{1}\right)
$$

for some $f \in k\left[x_{1}, z_{1}\right]$. In particular, using (15) and (7), and putting $x_{1}=0$ in (9), we have,

$$
P_{2}\left(0, \gamma z_{1}+\delta(0)\right)=\tau P_{1}\left(0, z_{1}\right)
$$

Now we have,

$$
y_{2}=\frac{P_{2}\left(x_{2}, z_{2}\right)}{x_{2}^{d}}=\frac{\tau P_{1}\left(x_{1}, z_{1}\right)+x_{1}^{d} f\left(x_{1}, z_{1}\right)}{\left(\lambda x_{1}\right)^{d}}=\nu y_{1}+g\left(x_{1}, z_{1}\right),
$$

where $\nu=\lambda^{-d} \tau \in k^{*}$ and $g=\lambda^{-d} f \in k\left[x_{1}, z_{1}\right]$. Therefore,

$$
k\left[x_{1}, y_{1}, z_{1}\right]=k\left[x_{2}, y_{2}, z_{2}\right] .
$$

We now show that $e_{1}=e_{2}$. Suppose, if possible, $e_{1}>e_{2}$. Using (77) and above, we have

$$
x_{1}^{e_{1}} B \cap k\left[x_{1}, y_{1}, z_{1}\right]=x_{2}^{e_{1}} B \cap k\left[x_{2}, y_{2}, z_{2}\right],
$$

i.e.,

$$
\left(x_{1}^{e_{1}}, Q_{1}\left(x_{1}, y_{1}, z_{1}\right)\right) k\left[x_{1}, y_{1}, z_{1}\right]=\left(x_{2}^{e_{1}}, x_{2}^{e_{1}-e_{2}} Q_{2}\left(x_{2}, y_{2}, z_{2}\right)\right) k\left[x_{2}, y_{2}, z_{2}\right] .
$$

Therefore, $Q_{1}\left(x_{1}, y_{1}, z_{1}\right) \in x_{2} k\left[x_{2}, y_{2}, z_{2}\right]=x_{1} k\left[x_{1}, y_{1}, z_{1}\right]$, which contradicts that $Q_{1}(X, Y, Z)$ is monic in $Y$. Hence, $e_{1} \leq e_{2}$ and by symmetry, we have

$$
e_{1}=e_{2}=e \text { say. }
$$

Thus, we have,

$$
x_{1}^{e} B \cap k\left[x_{1}, y_{1}, z_{1}\right]=x_{2}^{e} B \cap k\left[x_{2}, y_{2}, z_{2}\right],
$$

i.e.,

$$
\left(x_{1}^{e}, Q_{1}\left(x_{1}, y_{1}, z_{1}\right)\right) k\left[x_{1}, y_{1}, z_{1}\right]=\left(x_{2}^{e}, Q_{2}\left(x_{2}, y_{2}, z_{2}\right)\right) k\left[x_{2}, y_{2}, z_{2}\right] .
$$

Thus, $Q_{2}\left(x_{2}, y_{2}, z_{2}\right)=\kappa^{\prime} Q_{1}\left(x_{1}, y_{1}, z_{1}\right)+x_{1}^{e} h^{\prime}$ for some $\kappa^{\prime}, h^{\prime} \in k\left[x_{1}, y_{1}, z_{1}\right]$. Since $Q_{i}(X, Y, Z)$ 's are monic in $Y$, using (5) and (10), we see that

$$
s_{1}\left(=\operatorname{deg}_{Y} Q_{1}\right)=s_{2}\left(=\operatorname{deg}_{Y} Q_{2}\right)=s \text { say, }
$$


and $\kappa^{\prime} \equiv \nu^{s} \bmod x_{1}^{e} k\left[x_{1}, y_{1}, z_{1}\right]$. Let $\kappa=\nu^{s} \in k^{*}$. Replacing $\kappa^{\prime}$ by $\kappa$, we have,

$$
Q_{2}\left(x_{2}, y_{2}, z_{2}\right)=\kappa Q_{1}\left(x_{1}, y_{1}, z_{1}\right)+x_{1}^{e} h\left(x_{1}, y_{1}, z_{1}\right)
$$

for some $h \in k\left[x_{1}, y_{1}, z_{1}\right]$. In particular, using (5),(77),(10) and putting $x_{1}=0$ in (13), we have,

$$
Q_{2}\left(0, \nu y_{1}+g\left(0, z_{1}\right), \gamma z_{1}+\delta(0)\right)=\kappa Q_{1}\left(0, y_{1}, z_{1}\right)
$$

Hence,

$$
t_{2}=\frac{Q_{2}\left(x_{2}, y_{2}, z_{2}\right)}{x_{2}^{e}}=\frac{\kappa Q_{1}\left(x_{1}, y_{1}, z_{1}\right)+x_{1}^{e} h}{\left(\lambda x_{1}\right)^{e}}=\frac{\left(\kappa t_{1}+h\right)}{\lambda^{e}} .
$$

Conversely, suppose conditions (I) and (II) hold. Consider the $k$-algebra map $\phi: k[X, Y, Z, T] \rightarrow B_{1}$ defined by

$$
\begin{aligned}
\phi(X) & =\lambda x_{1} \\
\phi(Z) & =\gamma z_{1}+\delta\left(x_{1}\right) \\
\phi(Y) & =\nu y_{1}+g\left(x_{1}, z_{1}\right) \\
\phi(T) & =\theta t_{1}+h^{\prime \prime}\left(x_{1}, y_{1}, z_{1}\right)
\end{aligned}
$$

where $\nu=\lambda^{-d} \gamma^{r}, \theta=\lambda^{-e} \nu^{s}, g\left(x_{1}, z_{1}\right)=\lambda^{-d} f\left(x_{1}, z_{1}\right)$ and $h^{\prime \prime}\left(x_{1}, y_{1}, z_{1}\right)=\lambda^{-e} h\left(x_{1}, y_{1}, z_{1}\right)$. Then clearly,

$$
\phi\left(X^{d} Y-P_{2}(X, Z)\right)=\phi\left(X^{e} T-Q_{2}(X, Y, Z)\right)=0 .
$$

Thus $\phi$ induces a $k$-linear map $\bar{\phi}: B_{2} \rightarrow B_{1}$, which is surjective. Since both $B_{1}$ and $B_{2}$ are of the same dimension, we have $\bar{\phi}$ is an isomorphism.

It follows from the above result that no member of the family of double Danielewski surfaces is isomorphic to a member of the family of Danielewski surfaces.

Corollary 3.11. Let $A$ be any Danielewski surface, i.e., $A=\frac{k[X, Z, V]}{\left(X^{n} V-f(X, Z)\right)}$, where $n \geq 2, \operatorname{deg}_{Z} f(X, Z) \geq 2$ and $f(0, Z) \neq 0$. Let $B$ be a double Danielewski surface, i.e., $B$ be as in (1) with the parameters $r \geq 2$ and $s \geq 2$. Then $A$ is not isomorphic to $B$.

Proof. Let $f(X, Z)=f_{0}(Z)+X f_{1}(X, Z)$. Then the ring $A \cong A^{\prime}$, where

$$
A^{\prime}=k[X, Z, Y, V] /\left(X Y-f_{0}(Z), X^{n-1} V-Y-f_{1}(X, Z)\right) .
$$

Note that the ring $A^{\prime}$ is of the form in (11) with $\operatorname{deg}_{Y}\left(Y-f_{1}(X, Z)\right)=1$. Hence, by Theorem 3.10, $B \nsubseteq A^{\prime}$, as the $\operatorname{deg}_{Y} Q(X, Y, Z)=s \geq 2$.

Below we deduce a few properties of automorphisms of double Danielewski surfaces.

Theorem 3.12. Let $B$ be as in (1) and let the parameters $r, s$ and e satisfy the conditions (4) of Lemma 3.6. Let $R$ denote the subring $k[x, y, z]$ of $B$. Let $\psi \in \operatorname{Aut}_{\mathrm{k}}(B)$. Then:

(i) $\psi(k[x, z])=k[x, z]$.

(ii) $\psi(x)=\lambda x$ for some $\lambda \in k^{*}$.

(iii) $\psi\left(\left(x^{d}, P(x, z)\right) k[x, z]\right)=\left(x^{d}, P(x, z)\right) k[x, z]$. 
(iv) $\psi(k[x, y, z])=k[x, y, z]$.

(v) $\psi\left(\left(x^{e}, Q(x, y, z)\right) R\right)=\left(x^{e}, Q(x, y, z)\right) R$.

(vi) $\psi(t)=a t+b$, where $a \in k^{*}$ and $b \in R$.

Proof. Follows from the proof of Theorem 3.10 (see (6), (77), (8), (11), (12), (14)).

The next result gives a characterization of any automorphism of $B$.

Theorem 3.13. Let $B$ be as in (1) and let the parameters $r, s$ and e satisfy the conditions (4) of Lemma 3.6. Let $\psi$ be an endomorphism of $B$ satisfying (i) and (ii) of Theorem 3.12. Then $\psi$ is an automorphism of the ring $B$.

Proof. As $B$ is a Noetherian ring, it is enough to show that, $\psi(B)=B$. Since $B$ is generated by $x, y, z$ and $t$, by (i), it is enough to show that $y, t \in \psi(B)$.

Since $\psi(x)=\lambda x$ for some $\lambda \in k^{*}$ and $\psi(k[x, z])=k[x, z]$, we have $\psi(z)=\lambda_{2} z+\mu_{2}$ for some $\lambda_{2} \in k^{*}$ and $\mu_{2} \in k[x]$. Since $\psi$ is an endomorphism, we have $\psi\left(x^{d} y\right)=$ $\psi(P(x, z))$. Therefore,

$$
\begin{aligned}
\lambda^{d} x^{d} \psi(y) & =P(\psi(x), \psi(z)) \\
& =P\left(\lambda x, \lambda_{2} z+\mu_{2}\right) \\
& =\tau P(x, z)+f(x, z) \\
& =\tau x^{d} y+f(x, z),
\end{aligned}
$$

where $\tau=\lambda_{2}^{r} \in k^{*}, f(x, z) \in k[x, z]$ and $\operatorname{deg}_{Z} f(X, Z)<\operatorname{deg}_{Z} P(X, Z)=r$. From (15), we have $f(x, z) \in x^{d} B \cap k[x, z]=\left(x^{d}, P(x, z)\right) k[x, z]$. Since $\operatorname{deg}_{Z} f(X, Z)<$ $\operatorname{deg}_{Z} P(X, Z)$ and $P(X, Z)$ is monic in $Z$, it follows that $f(x, z)=x^{d} g(x, z)$ for some $g(x, z) \in k[x, z]$. Hence, from (15), we have

$$
y=\tau^{-1}\left(\lambda^{d} \psi(y)-g(x, z)\right) \in k[x, z, \psi(y)] \subseteq \psi(B) .
$$

Thus $k[x, y, z]=k[x, \psi(y), z]$. Now $\psi\left(x^{e} t\right)=\psi(Q(x, y, z))$, and hence

$$
\begin{aligned}
\lambda^{e} x^{e} \psi(t) & =Q(\psi(x), \psi(y), \psi(z)) \\
& =Q\left(\lambda x, \lambda^{-d}(\tau y+g(x, z)), \lambda_{2} z+\mu_{2}\right) \\
& =\nu Q(x, y, z)+h^{\prime}(x, y, z) \\
& =\nu x^{e} t+h^{\prime}(x, y, z),
\end{aligned}
$$

where $\nu=\left(\lambda^{-d} \tau\right)^{s}, h^{\prime}(x, y, z) \in k[x, y, z]$ and $\operatorname{deg}_{Y} h^{\prime}(X, Y, Z)<\operatorname{deg}_{Y} Q(X, Y, Z)=$ $s$. From (16), we have $h^{\prime}(x, y, z) \in x^{e} B \cap k[x, y, z]=\left(x^{e}, Q(x, y, z)\right) k[x, y, z]$. Since $\operatorname{deg}_{Y} h^{\prime}(X, Y, Z)<\operatorname{deg}_{Y} Q(X, Y, Z)$ and $Q(X, Y, Z)$ is monic in $Y$, we have $h^{\prime}(x, y, z)=$ $x^{e} h(x, y, z)$ for some $h(x, y, z) \in k[x, y, z]$. Hence, from (16), we have

$$
t=\nu^{-1}\left(\lambda^{e} \psi(t)-h(x, y, z)\right) \in k[x, y, z, \psi(t)] \subseteq \psi(B) .
$$

We now prove a stable isomorphism property of double Danielewski surfaces. 
Theorem 3.14. Let

$$
B_{d, e}=\frac{k[X, Y, Z, T]}{\left(X^{d} Y-P(X, Z), X^{e} T-Q(X, Y, Z)\right)},
$$

where $d, e \in \mathbb{N}, P(X, Z)$ is a monic polynomial in $Z$ with $\operatorname{deg}_{Z} P(X, Z)=r \geq 2$ and $Q(X, Y, Z)$ is a monic polynomial in $Y$ with $\operatorname{deg}_{Y} Q(X, Y, Z)=s \geq 2$. Let

$$
P(X, Z)=a_{0}(X)+a_{1}(X) Z+\cdots+a_{r-1}(X) Z^{r-1}+Z^{r},
$$

and

$$
Q(X, Y, Z)=b_{0}(X, Z)+b_{1}(X, Z) Y+\cdots+b_{s-1}(X, Z) Y^{s-1}+Y^{s} .
$$

Define

$$
P^{\prime}(X, Z):=a_{1}(X)+\cdots+(r-1) a_{r-1}(X) Z^{r-2}+r Z^{r-1}
$$

and

$$
Q^{\prime}(X, Y, Z)=b_{1}(X, Z)+2 b_{2}(X, Z) Y+\cdots+(s-1) b_{s-1}(X, Z) Y^{s-2}+s Y^{s-1} .
$$

Suppose that

$$
\left(P(0, Z), P^{\prime}(0, Z)\right) k[Z]=k[Z] \text { and }\left(P(0, Z), Q(0, Y, Z), Q^{\prime}(0, Y, Z)\right) k[Y, Z]=k[Y, Z] .
$$

Then, for $e \geq 2$,

$$
B_{d, e^{[1]}}^{[1} B_{d, e-1}^{[1]} .
$$

Proof. We write $B_{d, e}=B$ for notational convenience. As before, let $x, y, z$ and $t$ respectively denote the images of $X, Y, Z$ and $T$ in $B$. Let $\phi: B \rightarrow B[U]$ be an exponential map defined on $B$ by

$$
\begin{aligned}
\phi(x) & =x, \\
\phi(z) & =z+x^{d+e} U, \\
\phi(y) & =\frac{P\left(x, z+x^{d+e} U\right)}{x^{d}}=y+x^{e} U \alpha(x, z, U), \\
\phi(t) & =\frac{Q\left(x, y+x^{e} U \alpha(x, z, U), z+x^{d+e} U\right)}{x^{e}}=t+U \beta(x, y, z, U),
\end{aligned}
$$

where $\alpha(x, z, U) \in k[x, z, U], \beta(x, y, z, U) \in k[x, y, z, U]$. Let $A=B[w]=B^{[1]}$ and extend $\phi$ to $A$ by defining $\phi(w)=w-x U$. Let $f=x^{d+e-1} w+z$. Then $f \in A^{\phi}$. Now,

$$
P(x, f)-P(x, z)=x^{d+e-1}\left(P^{\prime}(0, z) w+x \theta\right)
$$

for some $\theta \in A$. Therefore,

$$
\begin{aligned}
P(x, f) & =P(x, z)+x^{d+e-1}\left(P^{\prime}(0, z) w+x \theta\right) \\
& =x^{d} y+x^{d+e-1}\left(P^{\prime}(0, z) w+x \theta\right) \\
& =x^{d} g,
\end{aligned}
$$


where $g=y+x^{e-1}\left(P^{\prime}(0, z) w+x \theta\right) \in A$. Note that, since $x^{d} g=P(x, f) \in A^{\phi}$ and $A^{\phi}$ is factorially closed in $A$ (cf. Lemma 2.1), we have $g \in A^{\phi}$. Now

$$
\begin{aligned}
Q(x, g, f) & =Q\left(x, y+x^{e-1}\left(P^{\prime}(0, z) w+x \theta\right), z+x^{d+e-1} w\right) \\
& =Q(x, y, z)+x^{e-1} P^{\prime}(0, z) Q^{\prime}(0, y, z) w+x^{e} \rho
\end{aligned}
$$

for some $\rho \in A$. Therefore,

$$
\begin{aligned}
Q(x, g, f) & =x^{e} t+(Q(x, g, f)-Q(x, y, z)) \\
& =x^{e} t+x^{e-1}\left(P^{\prime}(0, z) Q^{\prime}(0, y, z) w+x \rho\right) \\
& =x^{e-1}\left(P^{\prime}(0, z) Q^{\prime}(0, y, z) w+x t+x \rho\right) \\
& =x^{e-1} h
\end{aligned}
$$

where $h=P^{\prime}(0, z) Q^{\prime}(0, y, z) w+x t+x \rho \in A$. Note that, since $Q(x, g, f)=x^{e-1} h \in$ $A^{\phi}$ and $A^{\phi}$ is factorially closed in $A$, we have $h \in A^{\phi}$. From the given condition we have, $\left(Q(0, Y, Z), Q^{\prime}(0, Y, Z) P^{\prime}(0, Z), P(0, Z)\right) k[Y, Z]=k[Y, Z]$. Then there exist $a(Y, Z), b(Y, Z), c(Y, Z) \in k[Y, Z]$ such that

$$
Q^{\prime}(0, Y, Z) P^{\prime}(0, Z) a(Y, Z)+Q(0, Y, Z) b(Y, Z)+P(0, Z) c(Y, Z)=1 .
$$

Since $Q(0, y, z) \in x A$ and $P(0, z) \in x A$, we have,

$$
Q^{\prime}(0, y, z) P^{\prime}(0, z) a(y, z)+x \delta=1
$$

for some $\delta \in A$.

Let $v=\frac{w-a(g, f) h}{x}$. We first show that $v \in A$. Note that $a(g, f)-a(y, z) \in x A$. Let $a(g, f)-a(y, z)=x \gamma$ for some $\gamma \in A$. Now

$$
\begin{aligned}
w-h a(g, f) & =w-h a(y, z)-h(a(g, f)-a(y, z)) \\
& =w-h a(y, z)-h x \gamma \\
& =w-a(y, z)\left(P^{\prime}(0, z) Q^{\prime}(0, y, z) w+x t+x \rho\right)-h x \gamma \\
& =w\left(1-a(y, z) P^{\prime}(0, z) Q^{\prime}(0, y, z)\right)-x(a(y, z) t+a(y, z) \rho+h \gamma) \\
& =w x \delta-x(a(y, z) t+a(y, z) \rho+h \gamma) \in x A
\end{aligned}
$$

Thus, $v=\frac{w-h a(g, f)}{x} \in A$. Now, since $x, f, g, h \in A^{\phi}$, we have $\phi(v)=v-U$. Then, by Lemma 2.1(iii),

$$
A=A^{\phi}[v]=\left(A^{\phi}\right)^{[1]} .
$$

Let $E=k[x, f, g, h]$. Consider indeterminates $X, F, G$ and $H$ over $k$ so that $k[X, F, G, H]=$ $k^{[4]}$ and let

$$
E_{1}=\frac{k[X, F, G, H]}{\left(X^{d} G-P(X, F), X^{e-1} H-Q(X, G, F)\right)} \cong B_{d, e-1} .
$$

We first show that $E \cong E_{1}$. Clearly there exists a surjective $k$-algebra homomorphism $\Phi: k[X, F, G, H] \rightarrow E$ such that $\Phi(X)=x, \Phi(F)=f, \Phi(G)=g$ and $\Phi(H)=h$. Using (17) and (18), we see that $\Phi$ induces a surjective $k$-algebra map $\bar{\Phi}: E_{1} \rightarrow E$. 
Note that $A[1 / x]=E[1 / x][w]=E[1 / x]^{[1]}$ and hence dimension of $E$ is two. Therefore, as $E_{1}$ is an integral domain (cf. Lemma 3.3) of dimension $2, \bar{\Phi}$ is an isomorphism.

We now show that $A^{\phi}=E$. Clearly $E \subseteq A^{\phi}$. Since $A[1 / x]=E[1 / x][w]$, it follows that $E[1 / x]=A^{\phi}[1 / x]$. Therefore, to show that $E=A^{\phi}$, it is enough to show that $x E=x A^{\phi} \cap E$. Since $x A^{\phi}=x A \cap A^{\phi}$ by Lemma 2.1(i), it is enough to show that $x E=x A \cap E$, i.e., to show that the kernel of the map $\iota: E \rightarrow A / x A$ is $x E$. For $u \in A$, let $\tilde{u}$ denote the image of $u$ in $A / x A$. Note that

$$
A / x A=\frac{k[Y, Z, T, W]}{(P(0, Z), Q(0, Y, Z))}=\left(\frac{k[Y, Z]}{(P(0, Z), Q(0, Y, Z))}\right)[W, T]=k[\tilde{z}, \tilde{y}, \tilde{w}, \tilde{t}] .
$$

Clearly $\iota(f)=\tilde{z}, \iota(g)=\tilde{y}$ and $\iota(h)=P^{\prime}(0, \tilde{z}) Q^{\prime}(0, \tilde{y}, \tilde{z}) \tilde{w}$. Now by (19),$P^{\prime}(0, \tilde{z}) Q^{\prime}(0, \tilde{y}, \tilde{z}) \in$ $(A / x A)^{*}$. Hence, $\iota(E)=k[\tilde{z}, \tilde{y}, \tilde{w}]$ and $A / x A=\iota(E)[\tilde{t}]=\iota(E)^{[1]}$. Thus $\operatorname{dim} \iota(E)=$ $\operatorname{dim}(A / x A)-1=1$. Now as

$$
E / x E \cong E_{1} / x E_{1}=k[F, G, H] /(P(0, F), Q(0, G, F))=\left(\frac{k[F, G]}{(P(0, F), Q(0, G, F))}\right)^{[1]},
$$

we have $\operatorname{dim}(E / x E)=1=\operatorname{dim} \iota(E)$. Hence kernel of $\iota$ is $x E$. Thus $A^{\phi}=E$. Hence

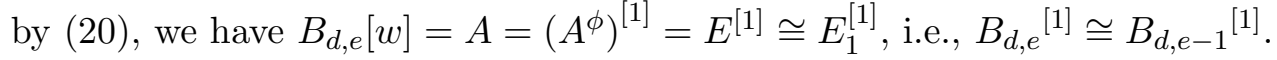

By Theorems 3.10 and 3.14, it follows:

Corollary 3.15. Under the hypotheses of Theorem 3.14, for every $d, e \in \mathbb{N}$,

$$
B_{d, e} ¥ B_{d, e+1} \text { but } B_{d, e^{[1]}}^{[1]} B_{d, e+1}{ }^{[1]} \text {. }
$$

Thus the rings $B_{d, e}$ provide counter-examples to the Cancellation Problem.

Acknowledgements. The authors thank Prof. Amartya Kumar Dutta for suggesting several changes to improve the exposition and simplifying the proofs. The first author acknowledges Department of Science and Technology for their SwarnaJayanti Fellowship. The second author acknowledges Council of Scientific and Industrial Research (CSIR) for their research grant.

\section{References}

[1] S. Abhyankar, P. Eakin, W. Heinzer, On the uniqueness of the coefficient ring in a polynomial ring, J. Algebra 23 (1972) 310-342.

[2] S.M. Bhatwadekar and N. Gupta, A Note on the Cancellation Property of $k[X, Y]$, J. Algebra and its Applications, (Special issue in honour of Professor Shreeram S. Abhyankar), 14(9) (2015)1540007(1-5).

[3] A.J. Crachiola, The hypersurface $x+x^{2} y+z^{2}+t^{3}=0$ over a field of arbitrary characteristic, Proc. Amer. Math. Soc. 134(5) (2005) 1289-1298.

[4] W. Danielewski, On a cancellation problem and automorphism groups of affine algebraic varieties, preprint 1989. 
[5] H. Derksen, O. Hadas and L. Makar-Limanov, Newton polytopes of invariants of additive group actions, J. Pure and Applied Algebra 156 (2001) 187-197.

[6] A.K. Dutta, N. Gupta and N. Onoda, Some patching results on algebras over two-dimensional factorial domains, J. Pure Appl. Algebra 216 (2012) 16671679 .

[7] K.H. Fieseler, On complex affine surfaces with $\mathbb{C}^{+}$-actions, Comment. Math. Helvetici 69 (1994) 5-27.

[8] Gene Freudenburg, Algebraic Theory of Locally Nilpotent Derivations(Second Edition), Encycl. Math. Sci., 136, Inv. Theory and Alg. Tr. Groups, VII, Springer-Verlag, (2017)

[9] T. Fujita, On Zariski problem, Proc. Japan Acad. 55A (1979) 106-110.

[10] N. Gupta, On the Cancellation Problem for the Affine Space $\mathbb{A}^{3}$ in characteristic p, Invent. Math. 195(1) (2014) 279-288.

[11] N. Gupta, On the family of affine threefolds $x^{m} y=F(x, z, t)$, Compositio Math. 150(6) (2014) 979-998.

[12] N. Gupta, On Zariski's Cancellation Problem in positive characteristic, Adv. Math. 264 (2014) 296-307.

[13] M. Hochster, Non-uniqueness of the ring of coefficients in a polynomial ring, Proc. Amer. Math. Soc. 34(1) (1972) 81-82.

[14] M. Miyanishi and T. Sugie, Affine surfaces containing cylinderlike open sets, J. Math. Kyoto Univ. 20 (1980) 11-42.

[15] P. Russell, On affine-ruled rational surfaces, Math. Ann. 255 (1981) 287-302. 